\section{Diagnostic role of anti-dsDNA antibodies: do not forget autoimmune hepatitis}

\section{Alessandro Granito, Luigi Muratori, Francesco Tovoli and Paolo Muratori}

In their Review (Pisetsky, D. S. \& Lipsky, P. E. New insights into the role of antinuclear antibodies in systemic lupus erythematosus. Nat. Rev. Rheumatol. 16, 565-579 (2020)) ${ }^{1}$, Pisetsky and Lipsky highlight the clinical role of antinuclear antibody (ANA) testing in patients with systemic lupus erythematosus (SLE) and properly state that anti-double-stranded DNA (dsDNA) antibodies are highly specific for the diagnosis of SLE; however, they refer to two papers that do not take into account the widely and historically recognized presence of these autoantibodies in individuals with autoimmune hepatitis ${ }^{2,3}$. In this regard, we would like to point out that knowledge of anti-dsDNA antibody positivity in autoimmune hepatitis dates back to 1956 when, because of the similarities to SLE, Mackay et al. proposed that this chronic liver disease be named 'lupoid hepatitis'.

The term lupoid hepatitis was first used to describe patients with chronic active hepatitis and positivity for the lupus erythematosus cell test, suggesting that these individuals have a specific form of liver disease that is often associated with extrahepatic complaints that are typically seen in SLE, including arthralgia and a cutaneous rash. In our opinion, the link between the two autoimmune diseases is of
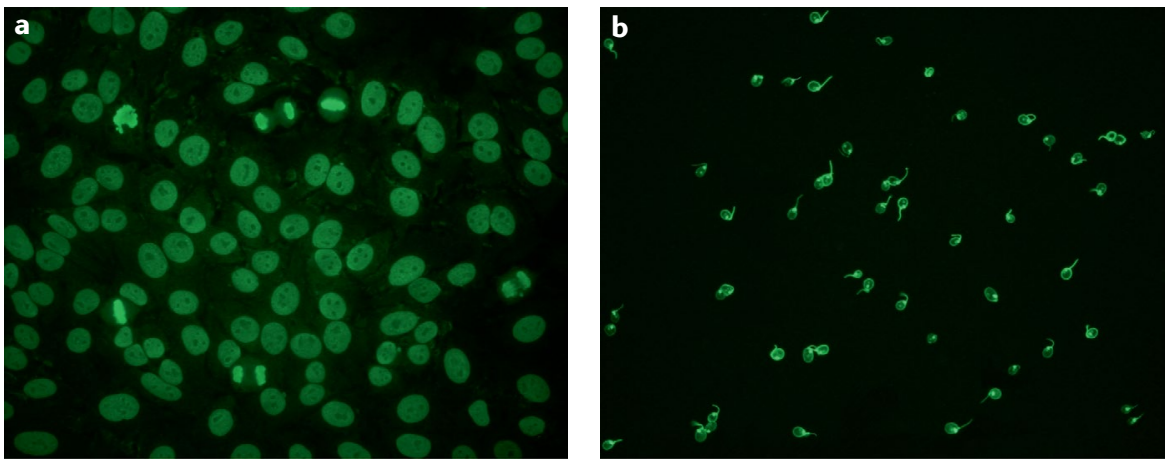

Fig. 1 | Antinuclear and anti-dsDNA antibody immunofluorescence patterns in autoimmune hepatitis. a | A typical autoimmune hepatitis antinuclear antibody staining pattern by indirect immunofluorescence in HEp-2 cells. A homogeneous fluorescence staining of the cell nuclei using a 1:40 dilution of serum from a patient with type 1 autoimmune hepatitis (serum titre 1:1,280). The condensed chromosomes of mitotic cells are positive, and the area surrounding the chromosomes is dark. $\mathbf{b} \mid$ Anti-double stranded DNA (dsDNA) antibody staining with serum from a patient with type 1 autoimmune hepatitis (titre 1:1,280). The pattern of positivity on Crithidia luciliae shows a strong kinetoplast staining (magnification 40x).
For the detection of anti-dsDNA antibodies, solid-phase immunoassays that use a mixture of multiple nuclear antigenic sources have lower specificity than immunofluorescence tests with Crithidia luciliae ${ }^{10}$, which contain high amounts of dsDNA in the kinetoplast and enable easy and simple interpretation of positive staining (FIG. 1 b).

We agree with Pisetsky and Lipsky that whoever orders an ANA test must be aware of which test will be used and, ideally, of its specificity and sensitivity; however, we would like to emphasize that in view of the potential clinical and immunological similarities between SLE and autoimmune hepatitis, a diagnosis of autoimmune hepatitis should always be considered in patients with ANA and anti-dsDNA antibody positivity.

There is a reply to this letter by Pisetsky, D. S. \& Lipsky, P. E. Nat. Rev. Rheumatol. https:// doi.org/10.1038/s41584-021-00574-6 (2020).

Alessandro Granito ${ }^{1,2,3 凶}$, Luigi Muratori ${ }^{1,2,3}$,
Francesco Tovoli,3 and Paolo Muratori $^{1,2,4}$
${ }^{1}$ Division of Internal Medicine, 'Division of Internal Medicine, Bologna, Italy.

${ }^{2}$ Center for the Study and Treatment of Autoimmune Diseases of the Liver and Biliary System, University of Bologna, Bologna, Italy. ${ }^{3}$ Department of Medical and Surgical Sciences, Alma Mater Studiorum, University of Bologna, Bologna, Italy.

${ }^{4}$ Department of the Science of Quality of Life, Alma Mater Studiorum, University of Bologna, Bologna, Italy.

凶e-mail: alessandro.granito@unibo.it https://doi.org/10.1038/s41584-021-00573-7

Pisetsky, D. S. \& Lipsky, P. E. New insights into the role of antinuclear antibodies in systemic lupus erythematosus. Nat. Rev. Rheumatol. 16, 565-579 (2020)

2. Pisetsky, D. S. Anti-DNA antibodies - quintessential biomarkers of SLE. Nat. Rev. Rheumatol. 12, 102-110 (2016).

3. Rekvig, O. P. The anti-DNA antibody: origin and impact, dogmas and controversies. Nat. Rev. Rheumatol. 11, dogmas and controve

Mackay, I. R., Taft, L. I. \& Cowling, D. C. Lupoid hepatitis. Lancet 271, 1323-1326 (1956).

European Association for the Study of the Liver. EASL Clinical Practice Guidelines: autoimmune hepatitis. J. Hepatol. 63, 971-1004 (2015).

6. Pappas, G., Granito, A. \& Bianchi, F. B. Systemic lupus erythematosus. N. Engl. J. Med. 358, 2412 (2008).

7. Muratori, P. et al. The serological profile of the autoimmune hepatitis/primary biliary cirrhosis overlap syndrome. Am. J. Gastroenterol. 104, 1420-1425 (2009).

8. Granito, A et al. Diagnosis and therapy of autoimmune hepatitis. Mini Rev. Med. Chem. 9, 847-860 (2009).

9. Chan, E. K. et al. Report of the first international consensus on standardized nomenclature of antinuclear antibody HEp-2 cell patterns 2014-2015. Front. Immunol. 6, 412 (2015).

10. Lange, C. E. et al. The kinetoplast immunofluorescence technic using Crithidia luciliae, a simple test for the detection of DNA-antibodies. Z. Hautkr. 52, 831-836 (1977).

Competing interests

The authors declare no competing interests. 